\title{
NEW OPTIMIZATION BASED METHOD FOR ESTIMATING PRIORITY WEIGHTS
}

\author{
Andrzej Z. Grzybowski \\ Institute of Mathematics, Czestochowa University of Technology \\ Czestochowa, Poland \\ andrzej.grzybowski@im.pcz.pl
}

\begin{abstract}
The estimation of priority vectors from pairwise comparison matrices is a core of the Analytic Hierarchy Process. Perhaps the most popular approach for deriving the priority weights is the right eigenvalue method (EM). Despite its popularity, various shortcomings of the EM have been described in literature. In this paper a new method for deriving priority vectors is proposed. This method makes use of the idea underlying the EM but in difference to the latter, the new one is optimization based. Important features of this new technique are studied via computer simulations and illustrated by some numerical examples.
\end{abstract}

Keywords: AHP, eigenvalue method, priority vector, constrained optimization

\section{Introduction}

In the AHP, pairwise comparisons of various alternatives are performed by the decision-maker (DM) and then the pairwise comparison matrix (PCM) is built. The elements of the matrix represent the DM judgments about the values of the priority ratios. Priority weights - assigned to each alternative and/or criterion - measure their relative importance. The weights form a so-called priority vector (PV). Generating PVs from the PCMs is the core of the AHP. In the early 1980s Saaty [1] suggested the right eigenvalue prioritization method (EM), that became the most popular method for deriving priority weights. During the last decades several other prioritization methods have been proposed in literature. However, each known method has its advantages and disadvantages. One can find in literature a number of papers devoted to comparative studies of various prioritization methods, [2-8]. Despite its popularity, the EM also has its share of criticism, see $[4,6,9-11]$. Some of its shortcomings will be addressed later on in more details.

This study proposes a new approach for deriving PVs. The basic concept is to combine Saaty's idea with some optimization procedures. As a result, we obtain estimation procedures which do not suffer from the above-mentioned EM's drawbacks and, moreover, provide us with naturally meaningful indicators of inconsistency of the PCMs. 
In the following section we state the prioritization problem formally. Next, in Section 2, the main prioritization approaches are briefly described. In Section 3 we introduce our proposals for deriving PVs. Section 4 presents the results of the simulation studies of the performance of the introduced methods as well as some numerical examples illustrating the advantages resulting from our approach. In Section 5 a problem connected with the COP is addressed.

\section{Formal statement of the prioritization problem}

A problem of deriving priority weights from PCM is to estimate a PV $\mathbf{w}=\left(w_{1}, \ldots, w_{n}\right)$ on the base of the matrix $\mathbf{A}=\left[a_{i j}\right]_{\mathrm{nxn}}$. Usually, the priority weights $w_{i}, i=1, \ldots, n$, are chosen to be positive and normalized to unity: $\sum_{i}^{n} w_{i}=1$. The elements $a_{i j}$ of the matrix $\mathbf{A}$ are the DM judgments about the priority ratios $w_{i} / w_{j}$, $i, j=1, \ldots, n$. The judgments are usually expressed in linguistic terms and then transformed into an appropriate numeric scale. A given PCM is said to be reciprocal (RPCM) if $a_{i j}=1 / a_{j i}$. PCM is called consistent if it is reciprocal and its elements satisfy the condition: $a_{i j} a_{j k}=a_{i k}$ for all $i, j, k=1, \ldots, n$. It is proved that a necessary and sufficient condition for a positive matrix $\mathbf{A}$ to be consistent is an existence of a unique PV $\mathbf{w}$ satisfying $a_{i j}=w_{i} / w_{j}$ for $i, j=1, \ldots, n$. PCM is said to be (ordinally) transitive if the following condition holds: (A) if for any $l=1, \ldots, n$ element $a_{l j}$ is not less than $a_{l k}$ then $a_{i j} \geq a_{i k}$ for $i=1, \ldots, n$ and (B) if for any $l=1, \ldots, n$ element $a_{j l}$ is not less than $a_{k l}$ then $a_{j i} \geq a_{k i}$ for $i=1, \ldots, n$.

It is obvious that in reality it cannot be expected that the elements of PCM give exact priority ratios. The evaluations of the ratios may depend on personal taste, experience, changes in one's knowledge, and may vary in time. One cannot also neglect rounding errors which can be quite big if we use discrete numeric scale, especially if it offers only a few values for consideration. Therefore in reality the PCM is typically inconsistent. Then the relation between the PCM elements and the priority weights can be expressed in the form

$$
a_{i j}=\varepsilon_{i j} \frac{w_{i}}{w_{j}}
$$

where $\varepsilon_{i j}$ is a perturbation factor which is expected to be near $1,[2,12,13]$. In the statistical approach and in various simulation studies the perturbation factor is interpreted as realization of a random variable.

In conventional AHP, the elements of PCM are collected only for the upper triangle of the matrix $\mathbf{A}$, and the remaining elements are computed as the inverse of the corresponding symmetric elements in the upper triangle that ensures the reciprocity of the PCM. This method of data collection artificially forces some consistency of judgments which is not always natural, see e.g. [2, 6, 10,11]. Some authors argue that enforcing this kind of consistency on the input data creates 
unnecessary dependency among observations and loses additional information contained perhaps in the elements of the lower triangle of $\mathbf{A}$ which may lead to poorer estimates of the priorities.

\section{Prioritization methods discussed in literature}

Choo et al. [2] discussed and compared 18 methods which may be used for deriving priority weights. These methods are derived from different concepts of the estimation quality criteria and under different assumptions about the perturbation factor structure. Except for the EM, most prioritization methods are optimization based. Such methods may assume a different criterion function and, consequently, result in different prioritization estimates. Among them the most popular is the logarithmic least squares method (also known as geometric mean method - GM) $[3,4,9]$. Imposing the normalization condition $\sum_{i=1}^{n} w_{i}=1$, the weights in GM can be estimated from the following formula:

$$
w_{i}=\left(\prod_{j=1}^{n} a_{i j}\right)^{1 / n} / \sum_{i=1}^{n}\left(\prod_{j=1}^{n} a_{i j}\right)^{1 / n}
$$

As it was already pointed out, the most commonly used prioritization method is the EM. This method is not optimization based. Let us describe the idea underlying EM in more detail. In a perfect judgment case, where there are no perturbations $\left(\varepsilon_{i j}=1\right)$ we have

$$
\mathbf{A w}=n \mathbf{w}
$$

Thus in this case the PV $\mathbf{w}$ can be calculated by solving the eigenvector equation (3). It turns out that for a consistent matrix $\mathbf{A}$ the number $n$ is the principal eigenvalue of $\mathbf{A}$, i.e. the largest solution of the characteristic equation: $\operatorname{det}(\mathbf{A}-\lambda \mathbf{I})=0$. It is also the only nonzero eigenvalue in this case. The relation (3) plays the main role in Saaty's approach. In the case where the matrix $\mathbf{A}$ is perturbed, the Saaty proposal is to use the normalized right eigenvector associated with the largest eigenvalue as an estimate of the true priority vector. Hence, to obtain the estimate we need to solve the general eigenvector equation

$$
\mathrm{Aw}=\lambda_{\max } \mathrm{W}
$$

where $\lambda_{\max }$ is the principal eigenvalue. For an arbitrary positive reciprocal matrix A the value $\lambda_{\max }$ is always real, unique and not smaller than $n$.

Apart from deriving priority vectors, another very important problem is how to measure the degree of inconsistence of the PCM. It is obvious that the significant violations of the consistency makes the inference useless (or at least questionable). 
In practice the only widely accepted rule of inconsistency measuring is due to Saaty and is closely related to EM. According to this concept the index $\mathrm{CI}(n)$ is given in the following form:

$$
\mathrm{CI}(n)=\frac{\lambda_{\max }-n}{n-1}
$$

The justification of the formula (5) as well as a more detailed description of the approach to consistency measuring can be found in various articles, see e.g. $[1,6$, 12]. However the index can hardly be interpreted in any intuitive way and is more and more often criticised and new indices which can be used for this purpose are proposed in literature, see $[6,14]$. What is more, the index $\mathrm{CI}(n)$ is useful only for RPCM and even then it can be very misleading, see e.g. [6] and references therein.

Although the EM has attracted much attention, especially in practical application, it has also been criticized in literature for several different reasons. In particular, some authors have pointed out that Saaty's procedure does not optimize any performance criterion. Thus it cannot be interpreted in statistical or optimization fashion and it is difficult to compare resulting PVs with the ones obtained with the help of other methods, see e.g. [3, 4, 9]. Moreover, unlike many optimization models, EM does not allow DM to introduce any additional constraints for the priority vector which, according to decision-maker opinions, should be satisfied by the weights, [6].

Another drawback of this method is that it should be used only for the so-called reciprocal PCMs, and as such it has a limited range of application. In real-world problems the reciprocity condition is artificially enforced by Saaty's method, and many authors argue that it would be much better if the DM would make all comparisons because then the PCM would contain more information about the unknown priority vector, see e.g. $[3,6,10,11]$. In the sequel a new prioritization method which does not suffer from these drawbacks is introduced. The performance of the new method will be compared with the performance of the most frequently recommend and used in AHP practice methods- the EM and GM, see [4, 8].

\section{Proposal of a new prioritization method}

In this proposal an idea of deriving the weights based on the relation (3) is adopted. However, in a new approach proposed in [5, 6], instead of solving the eigenvalue equation (4) one looks for a vector $\mathbf{w}$ which best approximates the relation (3), i.e. a vector for which

$$
\mathbf{A w} \cong n \mathbf{w}
$$

Consequently, as an estimate of the DM priorities, a vector $\mathbf{w}$ is used, which is a solution to the following optimization problem: 
subject to $\sum_{i=1}^{n} w_{i}=1, w_{i}>0, i=1, \ldots, n$. The function $D$ in (7) measures a distance between the vectors appearing on the right and left hand side of the relation (6).

Let $\mathbf{d}=\left(d_{1}, \ldots, d_{n}\right)^{\mathrm{T}}=\mathbf{A} \mathbf{w}-n \mathbf{w}$. There are various distance measures that can be used in () to derive the priorities. Some of them were discussed in [6] and [7]. Here we consider one of the most widely accepted distance measures, which appears in various optimization models. It is the sum of squared deviations $\sum_{i=1}^{n} d_{i}^{2}$. This measure leads to a prioritization method, which will be called in the sequel least squared deviation approximation (LSDA). To obtain the PV in this approach one needs to solve the following quadratic programming problem

$$
\min S S D(\mathbf{w})=\mathbf{w}^{\mathrm{T}} \mathbf{B} \mathbf{w}
$$

subject to

$$
\text { C1. } \sum_{j=1}^{n} w_{j}=1, \quad \text { and } \mathrm{C} 2 . \quad w_{i}>0, i=1, \ldots, n
$$

in the above model $\mathbf{B}=\left[(\mathbf{A}-n \mathbf{I})^{\mathrm{T}}(\mathbf{A}-n \mathbf{I})\right]$ with $\mathbf{I}$ being an identity matrix of order $n$.

It is easy to see that if the matrix $\mathbf{A}$ is inconsistent then the matrix $\mathbf{B}$ is nonsingular. In such a case we can find a closed-form formula for a vector $\mathbf{w}_{L S}$ minimizing (8) and satisfying the constraint $\mathrm{C} 1$. Using well-known solutions for minimizing a quadratic form under linear constraints, the following formula can be derived

$$
\mathbf{w}_{L S}=\mathbf{B}^{-1} \mathbf{e} /\left(\mathbf{e}^{\mathrm{T}} \mathbf{B}^{-1} \mathbf{e}\right)
$$

where $\mathbf{e}=(1, \ldots, 1)^{\mathrm{T}}$ is a column vector with all elements equal to 1 . Obviously, the vector given by (9) does not have to satisfy the positivity constraint C2. However, it is rarely the case if the PCM is both transitive and reciprocal. We have generated randomly 10000 such matrices (each of random order drawn from the interval $[4,12])$ and found only 16 examples when the vectors given by (9) have some negative coefficients. It is only $0.16 \%$ of all generated PCMs. The analogous percentage computed for PCMs which were transitive and nonreciprocal is greater and amounts to $12.7 \%$ while in the case of reciprocal and nontransitive PCMs the percentage equals $59.7 \%$ Obviously, in all such cases, we can use computer software to solve the minimization problem (8) numerically. One may notice that nowadays very efficient computer software is available for minimizing the quadratic form (8) under linear constraints of the type $\mathrm{C} 1$ and $\mathrm{C} 2$.

The existence of the closed-form formula (9) that allows to derive priorities in many practical problems is a very appealing future of the proposed method. It also has another important feature - easily interpretable consistency index. Let the 
minimum of (8) subject to constraints $\mathrm{C} 1$ and $\mathrm{C} 2$ be denoted as MSSD. Its value can be considered as an inconsistency measure. In a consistent case MSSD equals 0 . For inconsistent PCMs the index takes positive values. It is easy to note that the sum of the coefficients of the vector standing on the right hand side of the relation (6) is equal to $n$. Thus, if we divide MSSD by $n$, and take the square root of the result, then we obtain an index of inconsistency $\mathrm{II}_{\mathrm{LS}}=\sqrt{\mathrm{MSSD} / n}$. The value of $\mathrm{II}_{\mathrm{LS}}$ can be compared with 1, the total sum of priority weights. Roughly speaking, the value tells us what part of the total mass of weights has to be changed (added or subtracted dependently on the coefficient) to achieve equality in the formula (6).

Unlike the EM, the proposed prioritization method and resulting inconsistency index can be applied to any type of PCM and thus provide us with a tool for dealing with nonreciprocal PCMs. It is well known that in the case of nonreciprocal PCMs the index CI may take negative values. In such a case CI has no interpretation. In the next section we present an example of such a situation.

\section{Methods comparison via computer simulation - results and examples}

In this section we present some examples illustrating the usefulness of the proposed method as well as results of simulation comparison of the LSDA with EM and GM, the most popular and recommended methods, see [4, 8]. To compare the accuracy of the estimates obtained by the considered methods we simulate various situations related to various sources of the inconsistency of PCM. In the simulations we assume, similarly as in e.g. $[4,8,13]$, that we know the true PV. Next we generate an inconsistent PCM related to the known PV. The inconsistence is a result of various types of errors and/or perturbation factors. In literature various sources of inconsistence of the PCMs were named. The sources can be divided into two groups: errors resulting from the nature of human judgments and errors resulting from the technical realization of the comparison procedure. The errors from the second group are mainly the rounding errors and errors resulting from the forced reciprocity. The presence of the rounding errors is connected with the numerical ratio scale, whose values should be used by the DM to express its judgments, see e.g. $[11,12]$. In conventional AHP the most popular is Saaty numerical scale which consists of the integers 1 to 9 and their reciprocals. Possible problems resulting from the rounding errors illustrate the following example.

Example 1. Let the true PV be as follows: $\mathbf{w}=(0.691,0.173,0.126,0.010)^{\mathrm{T}}$. Then the approximated PCM (in the sequel denoted as APCM) containing the numbers from the Saaty scale which are the closest to the elements of the true ratio matrix is:

$$
\left[\begin{array}{cccc}
1 & 4 & 5 & 9 \\
1 / 4 & 1 & 1 & 9 \\
1 / 6 & 1 / 2 & 1 & 9 \\
1 / 9 & 1 / 9 & 1 / 9 & 1
\end{array}\right]
$$


It is worth noticing, that due to the rounding errors only, a nonreciprocal PCM is obtained. Indeed, for example the ratio $w_{1} / w_{3}$ equals 5.484 and the closest value taken from the ratio scale is 5 , but the ratio $w_{3} / w_{1}$ equals 0.182 and the closest value from the scale is $1 / 6$. Now, let us estimate the PV using the EM, GM and LSDA. We compare the results with the true PV. In these comparisons we use performance measures known from literature, see e.g. [2, 4, 8, 13]: the Pearson correlation coefficient $r$ between the estimated and true vectors, the Spearman rank correlation coefficient $\rho$, mean absolute deviation (MAD) and root-mean-squaredeviation (RMS). The two latter measures are given by the following formulae:

$$
\begin{gathered}
\operatorname{MAD}\left(\mathbf{w}, \mathbf{w}^{*}\right)=\frac{1}{n} \sum_{i=1}^{n}\left|w_{i}-w_{i}^{*}\right| \\
\operatorname{RMS}\left(\mathbf{w}, \mathbf{w}^{*}\right)=\sqrt{\frac{1}{n} \sum_{i=1}^{n}\left(w_{i}-w_{i}^{*}\right)^{2}}
\end{gathered}
$$

The results of the comparison are presented in Table 1. One can see that in this case the new method performs slightly better than the two others.

Table 1

Comparison of EM, GM and LSDA. Performance for rounding errors - Example 1

\begin{tabular}{|cccccc|}
\hline method & estimate & $r$ & $\rho$ & MAD & RMS \\
\hline EM & $(0.6067,0.1976,0.1615,0.0342)^{\mathrm{T}}$ & 0.9982 & 1 & 0.0422 & 0.0489 \\
GM & $(0.6158,0.1955,0.1582,0.0305)^{\mathrm{T}}$ & 0.9980 & 1 & 0.0408 & 0.0472 \\
LSDA & $(0.6158,0.1954,0.1581,0.0307)^{\mathrm{T}}$ & 0.9985 & 1 & 0.0376 & 0.0436 \\
\hline
\end{tabular}

Now let us address the problem of forced reciprocity. According to the conventional AHP setup, the elements of the lower triangle of the PCM should be computed as reciprocals of the appropriate elements from the upper triangle. Thus, in our example, the elements $a_{31}$ and $a_{32}$ should be changed to $1 / 5$ and 1, respectively. Results obtained for such a PCM are presented in Table 2.

Table 2

Comparison of EM, GM and LSDA. Performance for rounding errors and forced reciprocity - Example 1

\begin{tabular}{|cccccc|}
\hline method & estimate & $r$ & $\rho$ & MAD & RMS \\
\hline EM & $(0.5955,0.1894,0.1825,0.0326)^{\mathrm{T}}$ & 0.9951 & 1 & 0.0478 & 0.0572 \\
GM & $(0.6143,0.1843,0.1767,0.0247)^{\mathrm{T}}$ & 0.9935 & 1 & 0.0519 & 0.0619 \\
LSDA & $(0.6144,0.1841,0.1763,0.0252)^{\mathrm{T}}$ & 0.9961 & 1 & 0.0383 & 0.0468 \\
\hline
\end{tabular}

We see that the performance of all considered methods are now poorer than in the nonreciprocal case. It can also be observed in Tables 1 and 2 that the LSDA 
outperforms the other two methods with respect to all criteria. One may say that it is just an example and in other situations the ranking of the methods may be different. It appears, however, that the phenomena and relations observed in Example 1 are typical. In our studies we simulate a thousand such problems. In each case the number of alternatives $n$ is drawn from the set $\{4, \ldots, 12\}$ and then a random PV is generated for which we compute all performance characteristics described in Example 1. Table 3. presents the average results for PCMs with the rounding errors only (APCM) and for PCMs with rounding errors and forced reciprocity (FR-APCM).

Table 3

Comparison of EM, GM and LSDA. Performance for PCMs with rounding errors - average results for 1000 random PV

\begin{tabular}{|ccccccccc|}
\hline \multicolumn{9}{c}{ APCM } \\
\hline \multirow{2}{*}{ method } & $r$ & $\rho$ & MAD & RMS & $r$ & $\rho$ & MAD & RMS \\
\hline EM & 0.979 & 0.997 & 0.0120 & 0.0090 & 0.980 & 0.996 & 0.0136 & 0.0100 \\
GM & 0.978 & 0.996 & 0.0129 & 0.0095 & 0.980 & 0.995 & 0.0146 & 0.0106 \\
LSDA & 0.976 & 0.997 & 0.0114 & 0.0086 & 0.980 & 0.996 & 0.0123 & 0.0091 \\
\hline
\end{tabular}

Now let us consider the errors resulting from the nature of human judgments. They are often treated as realization of random variables and are commonly represented in the form (1), see e.g. [2, 6, 14]. Probability distributions (p.d.) of the perturbation factor $\varepsilon_{i j}$ mainly involve gamma, log-normal and uniform ones, [6, 13]. Following $[4,8]$ we consider three comparison frameworks that will be denoted $\mathrm{CF} 1, \mathrm{CF} 2$, and CF3. In CF1 the PCMs contain many small errors. In our simulations small error $\varepsilon_{i j}$, see (1), has p.d. that is uniform on the interval $[0.8,1.2]$. In the second comparison framework, the PCMs contain many small and one large error, in CF3 the PCMs contain many large errors. Large errors $\varepsilon_{i j}$ are generated according the p.d. having uniform distribution on the interval $[0.2,1.8]$.

Although we adopt the comparison approach described in $[4,8]$ we propose some changes. First, the simulations described in $[4,8]$ are based only on one priority vector. Moreover, the vector is not normalized and thus the observed average errors cannot be compared with errors corresponding to other vectors having different dimensions and priority values. To make the results more representative in our simulation we use 30 random normalized vectors having random dimensions. The dimension of each random vector is drawn from the set $\{4, \ldots, 12\}$. For each vector we generate 250 randomly disturbed PCMs with the perturbation factor having the p.d. dependent on the comparison framework, as described above. Similarly as in Example 1, we consider two types of approximation: with and without forced reciprocity. Next the performance measures are computed.

Another difference is that in our studies we also take into account the rounding errors. Therefore the randomly disturbed ratios are rounded to the closest values 
from Saaty's scale. Such simulation framework seems to be more realistic, because in the AHP procedure DM is always expected to express his/her opinions in a given scale. Table 4 presents the average results obtained in these studies.

Table 4

Comparison of EM, GM and LSDA under simulation frameworks CF1, CF2 and CF3

\begin{tabular}{|c|c|c|c|c|c|c|c|c|}
\hline \multicolumn{9}{|c|}{$\mathrm{CF} 1$ - average results for 30 random PVs } \\
\hline \multicolumn{4}{|c|}{ APCM } & \multicolumn{5}{|c|}{ FR-APCM } \\
\hline method & $r$ & $\rho$ & MAD & RMS & $r$ & $\rho$ & MAD & RMS \\
\hline EM & 0.972 & 0.932 & 0.0264 & 0.0203 & 0.993 & 0.973 & 0.0372 & 0.0284 \\
\hline GM & 0.966 & 0.922 & 0.0292 & 0.0225 & 0.991 & 0.973 & 0.0370 & 0.0283 \\
\hline LSDA & 0.971 & 0.930 & 0.0266 & 0.0204 & 0.993 & 0.973 & 0.0369 & 0.0283 \\
\hline \multicolumn{9}{|c|}{$\mathrm{CF} 2$ - average results for 30 random PVs } \\
\hline EM & 0.990 & 0.951 & 0.0225 & 0.0166 & 0.988 & 0.944 & 0.0243 & 0.0178 \\
\hline GM & 0.986 & 0.954 & 0.0239 & 0.0187 & 0.986 & 0.945 & 0.0259 & 0.0187 \\
\hline LSDA & 0.989 & 0.951 & 0.0214 & 0.0157 & 0.988 & 0.944 & 0.0224 & 0.0165 \\
\hline \multicolumn{9}{|c|}{ CF3 - average results for 30 random PVs } \\
\hline EM & 0.977 & 0.945 & 0.0239 & 0.0180 & 0.948 & 0.923 & 0.0337 & 0.0248 \\
\hline GM & 0.971 & 0.938 & 0.0266 & 0.0200 & 0.949 & 0.927 & 0.0341 & 0.0249 \\
\hline LSDA & 0.977 & 0.944 & 0.0237 & 0.0179 & 0.948 & 0.923 & 0.0325 & 0.0243 \\
\hline
\end{tabular}

All the presented results confirm that the methods EM, GM, and LSDA perform very similarly. However the LSDA seems to be the best method with respect to the values of average errors, both MAD and RMS. Taking into account these criteria, it demonstrates the best performance under 5 out of 6 simulation frameworks.

In Table 4, another interesting observation can be made. All considered methods perform significantly better in the case of the nonreciprocal matrices. This fact indicates the importance of making use of the nonreciprocal PCMs in AHP. In such a case, however, the Saaty's inconsistency index may be uninterpretable even in the case of slightly inconsistent PCMs resulting e.g. from the rounding errors. The problem is illustrated by the example below.

Example 2. Let the PCM be as follows:

$\left[\begin{array}{cccc}1 & 2 & 5 & 9 \\ 1 / 2 & 1 & 3 & 9 \\ 1 / 6 & 1 / 4 & 1 & 3 \\ 1 / 9 & 1 / 9 & 1 / 3 & 1\end{array}\right]$

We see that $a 31 \neq 1 / a 13$ and $a 32 \neq 1 / a 23$. However the PCM can hardly be considered as seriously inconsistent because, as we have already seen such perturbations can be observed even in the case of perfect judgments and may appear simply 
due to rounding errors. Indeed, if for example, the true ratios are $w_{1} / w_{3}=5.49$, and $w_{2} / w_{3}=3.45$ then the APCM will have values of the related elements as in our example. But, in this case, the Saaty inconsistency index CI equals -0.017 and, as negative, is uninterpretable, and that is why nonreciprocal PCMs are forbidden in conventional AHP. As we can see from the above simulation results, forced reciprocity often leads to the loss of quality of the resulting estimates. To avoid this drawback, one may use LSDA and the related index of inconsistency. In this case the index $\mathrm{II}_{\mathrm{LS}}$ is equal to 0.002 , and its small value indicates good consistency of the PCM. Roughly speaking, only less than $0.2 \%$ of the total mass of weights should be modified (added to or subtracted from the coefficients of the vector) to achieve the equality in (6). Indeed, based on such intuition, one may say that the inconsistency is not significant. However, in real world applications of the AHP, the DM needs a more precise and better justified threshold value which separates the acceptable judgment PCMs, and PCMs that should be rejected as "randomly generated". To obtain such thresholds, following Saaty's approach, let us study the empirical distribution of the values of $\mathrm{II}_{\mathrm{LS}}$ computed for random PCMs. This random index will be denoted by RII. Table 5 presents the statistical characteristics of empirical distributions obtained for RII generated for reciprocal PCM.

Table 5

Statistical characteristics of empirical distributions of RII(n) obtained for random reciprocal PCMs. For each number of alternatives $\boldsymbol{n}$ results are based on 10000 random reciprocal PCMs

\begin{tabular}{|c|c|c|c|c|c|c|c|c|}
\hline \multicolumn{2}{|c|}{$\begin{array}{c}\text { Empirical } \\
\text { distribution } \\
\text { characteristics }\end{array}$} & \multicolumn{7}{|c|}{ number of alternatives $n$} \\
\hline & & $n=4$ & $n=5$ & $n=6$ & $n=7$ & $n=8$ & $n=9$ & $n=10$ \\
\hline Mean & (MRII) & 0.239 & 0.282 & 0.307 & 0.320 & 0.325 & 0.321 & 0.323 \\
\hline \multirow{4}{*}{$p$-Quantiles } & $p=.01$ & 0.008 & 0.018 & 0.041 & 0.055 & 0.100 & 0.107 & 0.150 \\
\hline & $p=.05$ & 0.015 & 0.032 & 0.074 & 0.119 & 0.148 & 0.166 & 0.203 \\
\hline & $p=.10$ & 0.024 & 0.053 & 0.106 & 0.151 & 0.187 & 0.206 & 0.226 \\
\hline & $p=.15$ & 0.030 & 0.081 & 0.137 & 0.186 & 0.211 & 0.228 & 0.241 \\
\hline
\end{tabular}

However, the reciprocity is usually forced artificially, whilst the most traditional definition to characterize the consistency of PCMs is to use transitivity (see e.g. [10] and references in there) and intransitivity is prohibited by most theories. Thus in Table 6 results related to random PCMs with forced transitivity are also presented.

Adopting Saaty's approach for inference based on the new index, one may treat a given PCM as consistent enough if $\operatorname{II}_{\mathrm{LS}}(n)<t_{\alpha}(n)$, with $t_{\alpha}(n)=\alpha \operatorname{MRII}(n)$, for some prescribed consistency level $\alpha$. On the other hand, one may prefer to adopt the conventional statistical approach, and select a proper quantile of the empirical distribution of the corresponding RII. The order $p$ of the quantile should reflect the 
attitude of the DM towards the probability of accepting random PCM as a consistent one and may be interpreted as the required consistency level.

Table 6

Statistical characteristics of empirical distributions of RII $(n)$ obtained for random transitive PCMs. For each number of alternatives $n$ results are based on 10000 random transitive PCMs

\begin{tabular}{|c|c|c|c|c|c|c|c|c|}
\hline \multicolumn{2}{|c|}{$\begin{array}{c}\text { Empirical } \\
\text { distribution } \\
\text { characteristics }\end{array}$} & \multicolumn{7}{|c|}{ number of alternatives $n$} \\
\hline & & $n=4$ & $n=5$ & $n=6$ & $n=7$ & $n=8$ & $n=9$ & $n=10$ \\
\hline Mean & (MRII) & 0.055 & 0.040 & 0.029 & 0.026 & 0.022 & 0.018 & 0.016 \\
\hline \multirow{4}{*}{$p$-Quantiles } & $p=.01$ & .0007 & .0006 & .0006 & .0005 & .0003 & .0002 & .0002 \\
\hline & $p=.05$ & .0046 & .0029 & .0024 & .0021 & .0017 & .0015 & .0012 \\
\hline & $p=.10$ & .0086 & .0060 & .0046 & .0041 & .0034 & .0031 & .0027 \\
\hline & $p=.15$ & .0135 & .0088 & .0067 & .0061 & .0050 & .0043 & .0040 \\
\hline
\end{tabular}

For instance, let us consider Example 2. The PCM is transitive. It is well-known that very often DMs, based on their experience, assume that their judgments should be transitive and the transitivity is usually "forced" by the DM personal beliefs. Thus one may assume that it was filled in as such. Consequently, we should compare the index of inconsistency with the quantiles given in Table 6. If we assume the required consistency level equal to $p=0.05$, then the matrix from this example can be considered as the consistent one because ICS for this matrix equals 0.002 and the value is significantly less than 0.0046 - the quantile of order $p=0.05$ related to empirical distribution of RII(4). A more thorough discussion of the problem can be found in [6].

\section{Final remarks}

The approach proposed in this paper provides DM not only with a relatively simple prioritization technique, but also with intuitive and easily computable inconsistency indices. The simulation results described in Section 4 show that the new method, the LSDA, demonstrates similar or even better performance than EM or GM. However the advantage of the LSDA is that it can be used for both reciprocal and nonreciprocal PCMs because the related inconsistency index is naturally meaningful for all types of PCMs. As a optimization based technique the LSDA also allows the DM to implement various other conditions which, according to his/her opinions, should be satisfied by the weights. Such additional constraints based on a prior knowledge or resulting from the DM requirements can be easily included in the LSDA but not in the EM. An additional advantage of the LSDA is the simple closed-form formula (9) which can be often used for computing the PV. 


\section{References}

[1] Saaty T.L., Scaling method for priorities in hierarchical structures, J. Math. Psychol. 1977, 15, 3, 234-28.

[2] Basak I., Comparison of statistical procedures in analytic hierarchy process using a ranking test, Math. Comp. Model. 1998, 28, 105-118.

[3] Budescu D.V., Zwick R., Rapoport A., Comparison of the analytic hierarchy process and the geometric mean procedure for ratio scaling, Appl. Psychol. Meas. 1986, 10, 69-78.

[4] Choo E.U., Wedley W.C., A common framework for deriving preference values from pairwise comparison matrices, Comp. Oper. Res. 2004, 31, 893-908.

[5] Grzybowski A.Z., Goal Programming Approach for Deriving Priority Vectors - Some New Ideas, Scientific Research of the Institute of Mathematics and Computer Science 2010, 1(9), 17-27.

[6] Grzybowski A.Z., Note on a new optimization based approach for estimating priority weights and related consistency index, Expert Systems with Applications 2012, 39, 11699-11708.

[7] Kazibudzki P.T., Comparison of analytic hierarchy process and some new optimization procedures for ratio scaling, Scientific Research of the Institute of Mathematics and Computer Science 2011, 1(10), 101-108.

[8] Lin C-C., A revised framework for deriving preference values from pairwise comparison matrices, Euro. J. Oper. Res. 2007, 176, 1145-1150.

[9] Crawford G., Williams C.A., A note on the analysis of subjective judgment matrices, J. Math. Psychol. 1985, 29, 387-405.

[10] Hovanov N.V., Kolari J.W., Sokolov M.V., Deriving weights from general pairwise comparison matrices, Math. Soc. Sci. 2008, 55, 205-220.

[11] Lipovetsky S., Tishler A., Interval estimation of priorities in the AHP, Euro. J. Oper. Res. 114, 1997, 153-164.

[12] Saaty T.L., Decision making with the AHP Why is the principal eigenvector necessary, Euro. J. Oper. Res. 2003, 145, 85-91.

[13] Zahedi F., A simulation study of estimation methods in the analytic hierarchy process, SocioEcon. Plann. Sci. 1986, 20, 347-354.

[14] Koczkodaj W.W., A new definition of consistency of pairwise comparisons, Mathematical and computer modeling 1993, 18(7), 79-84.

[15] Saaty T.L., Vargas L.G. Comparison of eigenvalue, logarithmic least square and least square methods in estimating ratio, J. Math. Model. 1984, 5, 309-324. 